Virginia Gonfiantini; Franklin Marcelo Sánchez-Pástor; Alba Susana Valarezo-Cueva; Joe David Carpio-Rosales

\title{
El Buen Vivir y la Educación Superior Ecuatoriana desde la Complejidad Sistémica
}

\section{Good Living and Ecuadorian Higher Education from the Systemic Complexity}

\author{
Virginia Gonfiantini \\ vgonfiantini@multiversidadreal.edu.mx \\ Universidad Nacional de Rosario, Rosario \\ Argentina \\ https://orcid.org/0000-0002-0161-0973 \\ Franklin Marcelo Sánchez-Pástor \\ franklin.sanchez@unl.edu.ec \\ Universidad Nacional de Loja, Loja \\ Ecuador \\ http://orcid.org/0000-0002-4919-8631 \\ Alba Susana Valarezo-Cueva \\ alba.valarezo@unl.edu.ec \\ Universidad Nacional de Loja, Loja \\ Ecuador \\ https://orcid.org/0000-0002-5484-9232 \\ Joe David Carpio-Rosales \\ joe.carpio@unl.edu.ec \\ Universidad Nacional de Loja, Loja \\ Ecuador \\ https://orcid.org/0000-0001-6157-716X
}

Recibido: 15 de mayo de 2020

Revisado: 12 de junio de 2020

Aprobado: 22 de julio de 2020

Publicado: 01 de agosto de 2020 
Revista Arbitrada Interdisciplinaria KOINONIA

Año 2020. Vol V. N¹. Especial: Educación

Hecho el depósito de Ley: FA2016000010

ISSN: 2542-3088

FUNDACIÓN KOINONIA (F.K). Santa Ana de Coro. Venezuela.

Virginia Gonfiantini; Franklin Marcelo Sánchez-Pástor; Alba Susana Valarezo-Cueva; Joe David Carpio-Rosales

\title{
RESUMEN
}

El objetivo de la investigación consistió en analizar el buen vivir y la educación superior ecuatoriana desde la complejidad sistémica. Se desarrolló mediante una tipología descriptiva con diseño no experimental bibliográfico, lo cual permitió recopilar información de campo a 814 profesionales de la educación de la zona Sur del Ecuador. El $41 \%$ de los encuestados afirma que la organización curricular es siempre o casi siempre unidisciplinaria, desconectada del mundo, fragmentada, sin estructuras vinculantes entre los conocimientos de la ciencia, la profesión, el arte. Para que exista un adecuado proceso educativo desde el buen vivir y complejidad, debe desarrollarse un cambio paradigmático en la cosmovisión de los actores educativos, de ese modo, es posible trabajar en función de responder a las premisas estipuladas para tal fin, de lo contrario se corre el riesgo de seguir aplicando el paradigma tradicional por cuanto no genera cambios al estatus quo.

Descriptores: Experimento educacional; innovación educacional; reforma de la educación; democratización de la educación. (Palabras tomadas del Tesauro UNESCO).

\begin{abstract}
The objective of the research was to analyze the good living and Ecuadorian higher education from the systemic complexity. It was developed through a descriptive typology with a non-experimental bibliographic design, which made it possible to collect field information from 814 education professionals from the southern part of Ecuador. $41 \%$ of those surveyed affirm that the curricular organization is always or almost always unidisciplinary, disconnected from the world, fragmented, without binding structures between knowledge of science, profession, and art. For there to be an adequate educational process based on good living and complexity, a paradigmatic change must be developed in the worldview of educational actors, in this way, it is possible to work according to responding to the premises stipulated for this purpose, otherwise It runs the risk of continuing to apply the traditional paradigm because it does not generate changes to the status quo.
\end{abstract}

Descriptors: Educational experiments; educational innovations; educational reform; democratization of education. (Words taken from the UNESCO Thesaurus). 
Virginia Gonfiantini; Franklin Marcelo Sánchez-Pástor; Alba Susana Valarezo-Cueva; Joe David Carpio-Rosales

\section{INTRODUCCIÓN}

A partir de la promulgación de la Constitución del Ecuador del 2008, el país cambia su mirada y su modelo de desarrollo, adecuando un sistema educativo, que tiene por objeto construir la sociedad del Buen Vivir, este proceso de capacitación permitió enseñar un modelo en el cual se pretendía dejar de lado los saberes fragmentados, unidisciplinarias, para avanzar hacia la creación de conocimientos sistémicos, interdisciplinares, transdisciplinares, con conciencia humana y ética profesional, quedando pendiente la construcción de un Modelo Educativo y Pedagógico, ajustado a las premisas planteadas. Con la promulgación de la Ley Orgánica de Educación Superior (LOES) en el año 2010, el Ecuador inició un proceso dirigido a garantizar el derecho a la Educación Superior de calidad, la cual direccione hacia la excelencia, mediante su libre acceso, brindando estabilidad académica y permanencia en el sistema educativo sin discriminación alguna. Con la idea de fortalecer la educación y ofrecer una calidad académica de relevancia social, las Instituciones de Educación Superior han sido sometidas a un proceso de evaluación por parte del Consejo de Evaluación, Acreditación y Aseguramiento de la Calidad de la Educación Superior (CEAACES). Esta evaluación ha servido para concientizar sobre la calidad de educación ofertada a la sociedad, así como dar seguimiento al cumplimiento de los objetivos pedagógicos. Por otro lado, ha servido para iniciar la construcción de nuevos modelos de educación que contribuyan al mejoramiento significativo de las bases académicas de las instituciones de este nivel de educación (Gómez, et al., 2017).

Reconocida la necesidad de una nueva forma de educar, se modificó el currículo de las diferentes universidades del Ecuador para lograr su acreditación por parte de CEACEES y garantizar una educación de calidad. En algunos casos, esta modificación supuso reestructurar las mallas curriculares con base a los principios del pensamiento complejo, dando como resultado un nuevo currículo sistémico, el cual encamina a una ecologización de saberes. 


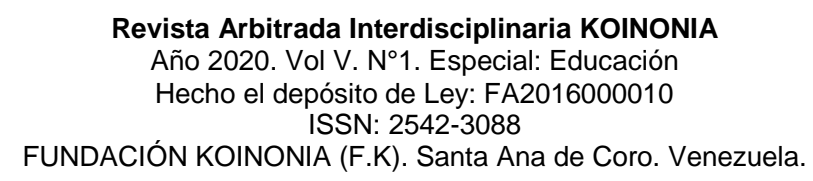

Virginia Gonfiantini; Franklin Marcelo Sánchez-Pástor; Alba Susana Valarezo-Cueva; Joe David Carpio-Rosales

En el Ecuador se apuesta por la educación desde el paradigma del Buen Vivir, modelo que fue inspirado en los pueblos indígenas del Sur de América (Arteaga-Cruz, 2017). No es un nuevo modelo de desarrollo, sino una alternativa al desarrollo. Tiene como prioridad la conexión de diversas culturas, teniendo en cuenta, por un lado, los saberes y principios del pasado, por el otro, las ciencias y las disciplinas contemporáneas, así se pretende transcender de la educación neoliberal que fue utilizado en las últimas décadas (ColladoRuano, 2017).

La educación en el Ecuador había estado al servicio del capitalismo, del racismo, la colonialidad, el poder y el patriarcalismo. Durante estos últimos años se ha tratado de hacer posible una educación que avance en su misión esencial tras el convencimiento de que toda persona es educable, en contra del decreto de la educación tradicional elitista del neoliberalismo, la cual ha evidenciado su naturaleza mercantil al servicio de las sociedades y culturas que normalizan la divergencia.

Para comprender, entrelazar e interrelacionar los diferentes constructos teóricos sobre una formación profesional basada en el Buen Vivir, es necesario apoyarse en la teoría del pensamiento complejo, la cual permite realizar conexiones entre diferentes saberes. Los estudiantes universitarios deben tener una formación interdisciplinaria. Este es uno de los aspectos más importantes en su formación académica debido a la diversidad de problemas en la sociedad que necesitan ser resueltos de una manera interdisciplinaria y eficaz.

Los futuros profesionales necesitan una formación básica en las diferentes áreas científicas, puesto que es imperativo para establecer relaciones de contenidos al momento de la práctica profesional. Mediante esto se puede lograr un aprendizaje básico, sistémico, sobre todo, contribuir a mejorar la calidad de vida en los diferentes niveles de la sociedad (Bustamante, Ayllón \& Escanés, 2018). Es por ello que se hace un llamado a repensar la educación, de tal forma que se concientice a la sociedad sobre la importancia de una pedagogía que permita resolver la diversidad de factores que van contribuyendo al agravamiento de problemas sociales, económicos, políticos y ambientales 
Virginia Gonfiantini; Franklin Marcelo Sánchez-Pástor; Alba Susana Valarezo-Cueva; Joe David Carpio-Rosales

Para comprender la incertidumbre que está inmersa en la formación docente, es necesario abordar nuevas teorías basadas en el pensamiento complejo, el buen vivir y las epistemologías del Sur (Aragón-Andrade, 2011). Son diferentes, pero se complementan unas con otras dando como resultado la construcción de conocimientos íntegros, duraderos, próximos a la realidad dinámica donde se desenvolverán los futuros profesionales.

Construir lineamientos alternativos sobre una propuesta educativa a partir de una visión planetaria, repensando el bien común, es necesario, es urgente, siempre considerando la identidad y el contexto en este caso el mantenido por el estado ecuatoriano a partir de la Constitución del 2008, con su filosofía del Buen Vivir, conscientes que existen duros golpes a aquellos proyectos históricos alternativos al neoliberalismo, al capitalismo, como bien lo han calificado muchos intelectuales del norte y del sur del planeta. El mismo Estado Social de Derecho, producto de la férrea actitud de los trabajadores, de sus continuas luchas, inicialmente dadas en la Europa de fines del siglo XIX e inicios del XX, pero que recorrieron también las calles de América, ha recibido los embates del neoliberalismo de las últimas décadas (Molina, 2020).

Se genera un debate sobre la construcción de otras vías de mejoramiento o desarrollo educativo, siendo por ello necesario realizar un diálogo de saberes: por un lado, el pensamiento complejo y por el otro el Buen Vivir. Estas dos formas de pensar nos proyectan hacia una ecologización del conocimiento, la cual deberá orientar la educación mediante una pedagogía auténtica, para el Buen Vivir. En función de lo planteado, el objetivo de la investigación consistió en analizar el buen vivir y la educación superior ecuatoriana desde la complejidad sistémica.

\section{MÉTODO}

La investigación se desarrolló mediante una tipología descriptiva con diseño no experimental bibliográfico, lo cual permitió recopilar información de campo a 814 profesionales de la educación de la zona Sur del Ecuador, mediante la aplicación de un 
Virginia Gonfiantini; Franklin Marcelo Sánchez-Pástor; Alba Susana Valarezo-Cueva; Joe David Carpio-Rosales

cuestionario en escalamiento de Likert de cinco alternativas de respuestas, aplicado mediante encuesta online, el instrumento fue validado mediante coeficiente de Alfa de Cronbach obteniéndose un coeficiente de 0,83 catalogándose como confiable para su aplicación, la información recopilada se procesó mediante estadística descriptiva.

\section{RESULTADOS}

El 31\% de los encuestados afirman que la operativización del currículo no se ha podido trabajar de forma efectiva todavía con un enfoque complejo, por cuanto esto demanda la interrelación y unificación de términos, conceptos, modelos, teorías y métodos, pero sobre todo de actitudes; mientras las autoridades educativas, no establezcan lineamientos claros y permanentes en relación al proceso de gestión del currículo en la Educación Superior desde el nuevo modelo de formación universitaria en Ecuador, no existirán mayores avances.

El $41 \%$ de los encuestados afirma que la organización curricular es siempre o casi siempre unidisciplinaria, desconectada del mundo, fragmentada, sin estructuras vinculantes entre los conocimientos de la ciencia, la profesión, el arte, la investigación, de los contextos, saberes y cultura, lo que afecta directamente en la consecución de los objetivos educativos nacionales.

\section{DISCUSIÓN}

La incongruencia entre el paradigma del Buen Vivir con la educación de los futuros profesionales radica en que mientras por un lado estamos obligados a responder a los grandes desafíos universales y a transformar la forma de pensar, sentir, hacer, por el otro también nos incentiva a llegar a un conocimiento riguroso, lógico e intercultural de este mundo que cada día se vuelve más profundo, complejo y lleno de incertidumbre (Rodríguez, et al. 2018). El Buen Vivir permite expandirnos a las culturas ancestrales aceptando el reto de la interculturalidad; pero esto es algo inútil sin mirar hacia los saberes tradicionales y ancestrales, sin juntar las ciencias a dichos saberes; pero, sobre 


\section{Virginia Gonfiantini; Franklin Marcelo Sánchez-Pástor; Alba Susana Valarezo-Cueva;} Joe David Carpio-Rosales

todo, sin entrelazar uniones con lo que se ha establecido en la incertidumbre de la vida, como algo imposible, impensable e impredecible (Fajardo, et al., 2019).

Para responder a la incertidumbre del presente con respecto al futuro tenemos que realizar miradas hacia el pasado, tratando de evitar la repetición, sobre todo, los propios del miedo frente a lo nuevo. En este sentido, el Buen Vivir no es un paradigma en retroceso, más bien es un cambio en la manera de comprender el tiempo, de experimentar, construir el espacio y apreciar la vida (Bravo, et al., 2019).

Para dar respuestas a los problemas actuales de la sociedad se necesita romper los paradigmas tradicionalistas y abordar las teorías de la complejidad, porque estas teorías se acercan a los problemas epistemológicos desde la propia ciencia, tomando como base los desarrollos científicos más recientes, y no solo desde la filosofía (Calvo-Cereijo, 2019). La complejidad propone una visión no lineal, no acumulativa del conocimiento científico; propone una complejización de conocimientos. Esta no presupone, de inmediato, una epistemología compleja; más bien, la epistemología compleja es un requisito para incorporar tal complejización (Chacón-Prado, 2015). Mediante esto se pretende reconocer y analizar los fenómenos multidimensionales en lugar de aislarlos, mutilando cada una de sus dimensiones (Borroto-López, 2015).

De allí que Gómez et al. (2016) considera que "la complejidad en Morín no trata de los asuntos propios de las así llamadas ciencias de la complejidad, sino de un nuevo horizonte epistemológico para organizar el pensamiento" (p. 473). Estos horizontes epistemológicos pretenden dotarnos de conocimientos que posibiliten la emergencia de una nueva manera de ser, pensar, actuar y convivir, posibilitando una transformación en la sociedad.

La epistemología compleja en la construcción de conocimiento gira alrededor del problema de la verdad, pasando de perspectiva en perspectiva, de verdades parciales, intentando efectuar una rearticulación del saber, del conocimiento inseparable de una reflexión fundamental (Juárez \& Comboni-Salinas, 2012). Para mantener abierta la problemática de la verdad, considera cualquier conocimiento, ya sea epistémico o extra 
Virginia Gonfiantini; Franklin Marcelo Sánchez-Pástor; Alba Susana Valarezo-Cueva; Joe David Carpio-Rosales

epistémico que cree verdadero, cualquier presunción de conocimiento, incluyendo el error, la ilusión, el desconocimiento. (Torrealba et al., 2018). Una educación auténtica no puede privilegiar la abstracción en el conocimiento, debe enseñar a contextualizar, concretar y globalizar, la educación transdisciplinaria reevalúa el rol de la intuición, del imaginario, de la sensibilidad y del cuerpo en la transmisión de los conocimientos (Sanabria-González, 2019).

Para que exista un adecuado proceso educativo desde el buen vivir y complejidad, debe desarrollarse un cambio paradigmático en la cosmovisión de los actores educativos, de ese modo, es posible trabajar en función de responder a las premisas estipuladas para tal fin, de lo contrario se corre el riesgo de seguir aplicando el paradigma tradicional por cuanto no genera cambios al estatus quo, efectuándose una mezcla pedagógica de complejidad y racionalidad, generando confusión en la práctica educativa, aunado a la pérdida de imagen, desconfianza, lo cual terminará por afianzar los hábitos cotidianos escolares.

El sector universitario del Ecuador debe generar una profunda reflexión sobre su rol y devenir en la sociedad de cara a los próximos años, por cuanto esto permite establecer el debate sobre la formación necesaria para transcender a niveles de progreso en función de las premisas del buen vivir, sin embargo, el debate debe ser despolitizado con la finalidad de comprender en profundidad la esencia de los paradigmas emergentes en la generación de una cultura científica donde se pueda integrar asertivamente los saberes ancestrales y cientificistas.

Siendo generar una educación transdisciplinar en razón de reconstituir el sistema educativo en función de abordar la realidad desde múltiples vertientes sinérgicas en razón de comprender desde una óptica científica en razón de perspectivas investigativas donde se confluya en la posibilidad de contar con un conocimiento sistémico afrontando los retos sociales y tecnológicos del mundo global en razón de compaginar la formación universitaria no con un mercado, sino, como un factor intelectual e intercultural de una neo epistemología social (Collado-Ruano, et al., 2018). 
Virginia Gonfiantini; Franklin Marcelo Sánchez-Pástor; Alba Susana Valarezo-Cueva; Joe David Carpio-Rosales

\section{CONCLUSIÓN}

En el currículo universitario ecuatoriano existen deficiencias que no permiten generan efectivamente aprendizajes desde los principios epistémicos de la complejidad, que orientan la formación profesional del diálogo de saberes y la interculturalidad, en el cumplimiento de los objetivos del Buen Vivir; esto lo manifiestan el $41 \%$ de las autoridades académicas encuestadas. En el modelo educativo, la realidad de la educación superior ecuatoriana no es entendida desde una mirada sistémica y holista, por cuanto la gestión curricular debería promoverse desde la incertidumbre, como principio de la complejidad sistémica, siendo necesaria para abordar la realidad desde una óptica compleja, es decir, lo cual contrasta con el paradigma de la veracidad científica. Se devela la necesidad de generar una transición paradigmática del modelo tradicional hacia el complejo y del buen vivir, por cuanto al no existir un empoderamiento por parte de los actores educativos de las premisas filosóficas de ambos enfoques, será difícil lograr concretar una educación tal como se presentan en los postulados epistemológicos del buen vivir y complejidad.

\section{FINANCIAMIENTO}

No monetario

\section{AGRADECIMIENTO}

A la Universidad Nacional de Loja, por su apoyo y estímulo en el desarrollo de la investigación. 
Revista Arbitrada Interdisciplinaria KOINONIA

Año 2020. Vol V. N¹. Especial: Educación

Hecho el depósito de Ley: FA2016000010

ISSN: 2542-3088

FUNDACIÓN KOINONIA (F.K). Santa Ana de Coro. Venezuela.

Virginia Gonfiantini; Franklin Marcelo Sánchez-Pástor; Alba Susana Valarezo-Cueva;
Joe David Carpio-Rosales

\section{REFERENCIAS CONSULTADAS}

Aragón-Andrade, O. (2011). Una epistemología del Sur: La reinvención del conocimiento y la emancipación social. [An Epistemology of the South: The Reinvention of Knowledge and Social Emancipation]. Alteridades, 21(41), 181-184.

Arteaga-Cruz, E. (2017). Buen Vivir (Sumak Kawsay): definiciones, crítica e implicaciones en la planificación del desarrollo en Ecuador. [Good Living (Sumak Kawsay): definitions, critique and implications for development planning in Ecuador]. Saúde em Debate, 41(114), 907-919. https://doi.org/10.1590/0103-1104201711419

Borroto-López, L. (2015). Conocimiento, pensamiento complejo y universidad. [Knowlwdge, complex thinking, and university]. Revista Cubana de Educación Superior, 34(2), 28-33.

Bravo, L., Zea Barahona, C., Manuel Gorozabel, V., \& Carolina Moreira, D. (2019). Fuerza laboral femenina del sector industrial pesquero en el Sumak Kawsay (buen vivir) ecuatoriano. [Female labor force of the fishing industrial sector in the Ecuadorian Sumak Kawsay (good living)]. CIENCIAMATRIA, 6(10), 307-325. https://doi.org/10.35381/cm.v6i10.132

Bustamante, L., Ayllón, S., \& Escanés, G. (2018). Abordando la trayectoria universitaria desde el pensamiento complejo. [Addressing the educational paths from Complex Thought]. Praxis Educativa, 22(3), 64-70.

Calvo-Cereijo, M. (2019). Pensamiento complejo y transdisciplina. [Complex thinking and transdiscipline]. Sophia, Colección de Filosofía de la Educación, (26), 307-326.

Chacón-Prado, M. de J. (2015). Congruencia del pensamiento complejo de Edgar Morín en la metodología de la educación a distancia. [Congruence of Edgar Morín's Complex Thought in Distance Education Methodology]. Revista Espiga, 14(30), 75-82. https://doi.org/10.22458/re.v14i30.933

Collado-Ruano, J, Madroñero-Morillo, M, \& Álvarez-González, F. (2018). Educación transdisciplinar: formando en competencias para el buen vivir. [Transdisciplinary education: training in skills for the good living]. Ensaio: Avaliação e Políticas Públicas em Educação, 26(100), 619-644. https://doi.org/10.1590/s0104$\underline{40362018002601487}$ 
Virginia Gonfiantini; Franklin Marcelo Sánchez-Pástor; Alba Susana Valarezo-Cueva; Joe David Carpio-Rosales

Collado-Ruano, J. (2017). Interculturalidad y descolonialidad: Retos y desafíos epistemológicos; Interculturalidad e descolonialidad: desafíos epistemológicos. [Interculturality and Decoloniality: Epistemological Challenges]. Revista nuestrAmérica, 5(9), 38-57.

Fajardo, Z., Colcha Beltrón, R., Balladares Torres, J., \& Madrid, E. (2019). Análisis comparativo de los estudiantes superdotados del Ecuador y Perú. [Comparative analysis of gifted students from Ecuador and Peru]. Revista Arbitrada Interdisciplinaria Koinonía, 4(8), 710-721. http://dx.doi.org/10.35381/r.k.v4i8.379

Gómez, V, Tolozano, M, \& Delgado, N. (2017). La Acreditación Institucional de la Calidad en los Institutos Superiores Técnicos y Tecnológicos del Ecuador desde la Perspectiva de un Instituto Acreditado. [The Institutional Accreditation of Quality in Technical and Technological Institutes of Higher Education in Ecuador from the Perspective of an Accredited Institute]. Formación universitaria, 10(6), 59-66. https://dx.doi.org/10.4067/S0718-50062017000600007

Gómez-Arrijos, C, Hernández-Hernández, M, Ramos-Sánchez, R. (2016). Principios epistemológicos para la enseñanza aprendizaje, según el pensamiento complejo de Edgar Morín. [Epistemological principles for the teachinglearning process, according to the educational thought of Edgar Morin]. Pueblo Continente, 27(2), p. 471-479.

Hidalgo-Capitán, A, \& Cubillo-Guevara, A. (2018). Orto y ocaso del buen vivir en la planificación nacional del desarrollo en Ecuador (2007-2021). [Rise and Decline of Good Living in the National Development Planning in Ecuador (2007-2021)]. 78, 37-54.

Juárez, J, \& Comboni-Salinas, S. (2012). Epistemología del pensamiento complejo. Reencuentro. [Epistemology of complex thought. Reunion]. Análisis de Problemas Universitarios, (65),38-51.

Molina, M. (2020). Visión descolonizadora de la gerencia transformacional en los circuitos educativos. [Decolonizing vision of transformational management in educational circuits]. $\quad$ EPISTEME KOINONIA, $\quad 3(5), \quad$ 88-101. http://dx.doi.org/10.35381/e.k.v3i5.532

Rodríguez, M, Aguilar, J, \& Apolo, D. (2018). El Buen vivir como desafío en la formación de maestros: aproximaciones desde la Universidad Nacional de Educación del Ecuador. [The Challenge of Good Living in Teacher Education: Approximations from Universidad Nacional de Educación in Ecuador]. Revista mexicana de investigación educativa, 23(77), 577-596. 
Revista Arbitrada Interdisciplinaria KOINONIA

Año 2020. Vol V. N¹. Especial: Educación

Hecho el depósito de Ley: FA2016000010

ISSN: 2542-3088

FUNDACIÓN KOINONIA (F.K). Santa Ana de Coro. Venezuela.

Virginia Gonfiantini; Franklin Marcelo Sánchez-Pástor; Alba Susana Valarezo-Cueva; Joe David Carpio-Rosales

Sanabria-González, J. (2019). Ciencias de la complejidad y pensamiento complejo en psicoterapia. Una revisión. [Sciences of complexity and complex thinking in psychotherapy. A review]. Tesis Psicológica, 14(1), 82-101. https://doi.org/10.37511/tesis.v14n1a5

Torrealba, C, Pérez, P, Castillo, N. (2018). El pensamiento complejo y la transcomplejidad: visión emergente en el desarrollo de un enfoque epistemológico en la investigación educativa. [Complex thinking and transcomplexity: emergent vision in the development of an epistemological approach in educational research]. Revista Arbitrada del Centro de Investigación y Estudios Gerenciales. Número 33, 139-154.

C2020 por los autores. Este artículo es de acceso abierto y distribuido según los términos y condiciones de la licencia Creative Commons Atribución-NoComercial-Compartirlgual 4.0 Internacional (CC BY-NC-SA 4.0) (https://creativecommons.org/licenses/by-nc-sa/4.0/). 